\title{
COMMENTARY
}

\section{Lest we forget the endothelial glycocalyx in sepsis}

Anne Burke-Gaffney ${ }^{* 1}$ and Timothy W Evans ${ }^{1,2}$

\begin{abstract}
Sepsis is the third largest cause of death in industrialised countries, but treatment remains largely supportive and effective therapeutic interventions are urgently needed. Disruption and dysfunction of the microvascular endothelium leading directly or indirectly to multiple organ failure are now recognised to underpin the pathophysiology of sepsis. Biomarkers of endothelial activation may therefore assume an important role in guiding future research efforts. We suggest that integral to this approach is the investigation and evaluation of endothelial glycocalyx biomarkers, not only as indicators of the pathogenic process but also to inform the development of pharmacological and other therapies.
\end{abstract}

The potential relevance of endothelial activation biomarkers to sepsis has been raised in both this journal and others [1-3]. Biomarkers for sepsis associated with the endothelial glycocalyx remain relatively unknown, however, and this commentary attempts to reverse this omission.

The term glycocalyx (sweet husk) was introduced 50 years ago to describe an extracellular polysaccharide coating of cells. Whilst electron microscopy revealed that the luminal surface of the endothelium expressed this structure, it was thought to be of little consequence or functional significance. What has become increasingly evident, however, is that the glycocalyx - now estimated to extend up to $1 \mu \mathrm{m}$ from the endothelial cell membrane represents a substantial intravascular compartment contributing significantly to vascular wall homeostasis [4]. Specifically, roles of the glycocalyx include maintenance of the vascular permeability barrier, mediation of shearstress-dependent nitric oxide production, and housing

*Correspondence: a.burke-gaffney@imperial.ac.uk

'Unit of Critical Care, Respiratory Science, National Heart and Lung Institute

Division, Faculty of Medicine Imperial College London, Dovehouse Street, London SW3 6LY, UK

Full list of author information is available at the end of the article vascular protective enzymes (for example, superoxide dismutase) and a wide array of coagulation inhibition factors such as antithrombin, the protein $\mathrm{C}$ system and tissue factor pathway inhibitor. The glycocalyx also modulates the inflammatory response by preventing leukocyte adhesion and binding numerous ligands, including chemokines, cytokines and growth factors [4-6].

Negatively charged and with a mesh-like structure, the endothelial glycocalyx is comprised of glycoproteins, proteoglycans, glycosaminoglycans (GAGs) and associated plasma proteins including albumin. Proteoglycans consisting of a core membrane-bound protein of the syndecan or glypican families with attached heparan or chondroitin sulphate GAG side chains are a prominent feature [6]. Hyaluronan - a nonsulphated, uncharged GAG with water-retaining properties - is attached or adsorbed onto other cell-surface anchored proteins (for example, CD44) and helps to stabilise the glycocalyx structure [7]. Alteration in the composition of the glycocalyx following exposure to an inflammatory insult is one of the earliest features of endothelial activation. It is now accepted that TNF $\alpha$, oxidised lipoproteins, lipopolysaccharide, thrombin, ischaemia/reperfusion, hyperglycaemia and growth factors all cause glycocalyx disruption via the action of proteases $[7,8]$ - leading either to partial degradation with release of GAG side chains, or to more severe damage characterised by shedding of core proteins [9].

Several studies have evaluated circulating levels of syndecan-1 and GAGs in patients with sepsis [10-13]. Plasma GAG levels were higher in patients with septic shock than in matched controls, and were significantly higher in nonsurvivors [10]. In the same study, syndecan-1 levels were also increased and correlated with the Sequential Organ Failure Assessment score [10]. In an additional study of 150 patients either with severe sepsis or septic shock or post-abdominal surgery without the systemic inflammatory response syndrome and healthy volunteers, significant increases in plasma syndecan-1 and heparan sulphate were observed in the sepsis and surgery groups. The highest syndecan-1 levels were detected in patients with sepsis and correlated with those of IL-6 [12]. A further study showed greater syndecan-1 levels in patients with septic shock compared with healthy controls, together with a positive correlation with 
vascular adhesion protein-1 and with day 1 Sequential Organ Failure Assessment scores [11]. Finally, hyaluronan levels, in addition to those of syndecan-1 and heparan sulphate, have been shown to increase with severity of sepsis [13].

Whilst the care of patients with sepsis has improved over the last decade, treatment remains largely supportive. The failure of two promising drugs, eritoran tetrasodium and drotrecogin alfa, to confer significant reduction in mortality suggests that novel approaches to sepsis research are required [14]. Given the fundamental, but perhaps relatively overlooked, role of the endothelial glycocalyx in regulating vascular integrity and functions central to the pathophysiology of sepsis, identifying interventions aimed at protecting or repairing it might prove a promising therapeutic target. Some clinically established therapies used for the treatment of sepsis (such as glucose control and steroid administration) and also approaches used in experimental studies (such as TNF $\alpha$ inhibition, antithrombin III, infusion of albumin and avoidance of natriuretic peptide release) are known to reduce glycocalyx disruption. However, drugs that might specifically increase the synthesis of glycocalyx components, refurbish the glycocalyx or selectively prevent protease degradation are not currently available $[7,15]$.

Future endeavours in the field of sepsis research, which are urgently required, should not only include components of the endothelial glycocalyx in the list of biomarkers, but also consider their potential as therapeutic targets for the development of new therapies.

\section{Abbreviations}

GAG, glycosaminoglycan; IL, interleukin; TNF, tumour necrosis factor.

\section{Competing interests}

The authors declare that they have no competing interests.

\section{Author details}

'Unit of Critical Care, Respiratory Science, National Heart and Lung Institute Division, Faculty of Medicine Imperial College London, Dovehouse Street, London SW3 6LY, UK. ${ }^{2}$ NIHR Respiratory Disease Biomedical Research Unit, Royal Brompton and Harefield NHS Trust Foundation Trust, London SW3 6NP, UK.
Published: 30 March 2012

\section{References}

1. Xing K, Murthy S, Liles WC, Singh JM: Clinical utility of biomarkers of endothelial activation in sepsis - a systematic review. Crit Care 2012, 16:R7

2. Paulus $P$, Jennewein C, Zacharowski K: Biomarkers of endothelial dysfunction: can they help us deciphering systemic inflammation and sepsis? Biomarkers 2011, 16(Suppl 1):S11-S21.

3. Pierrakos C, Vincent JL: Sepsis biomarkers: a review. Crit Care 2010, 14:R15

4. Van Teeffelen JW, Brands J, Stroes ES, Vink H: Endothelial glycocalyx: sweet shield of blood vessels. Trends Cardiovasc Med 2007, 17:101-105.

5. Choi Y, Chung H, Jung H, Couchman JR, Oh ES: Syndecans as cell surface receptors: unique structure equates with functional diversity. Matrix Biol 2011, 30:93-99.

6. Nieuwdorp M, Meuwese MC, Vink H, Hoekstra JB, Kastelein JJ, Stroes ES: The endothelial glycocalyx: a potential barrier between health and vascular disease. Curr Opin Lipido/ 2005, 16:507-511.

7. Broekhuizen LN, Mooij HL, Kastelein JJ, Stroes ES, Vink H, Nieuwdorp M: Endothelial glycocalyx as potential diagnostic and therapeutic target in cardiovascular disease. Curr Opin Lipido/ 2009, 20:57-62.

8. Chappell D, Westphal M, Jacob M: The impact of the glycocalyx on microcirculatory oxygen distribution in critical illness. Curr Opin Anaesthesiol 2009, 22:155-162.

9. Manon-Jensen T, Itoh Y, Couchman JR: Proteoglycans in health and disease: the multiple roles of syndecan shedding. FEBS J 2010, 277:3876-3889.

10. Nelson A, Berkestedt I, Schmidtchen A, Ljunggren L, Bodelsson M: Increased levels of glycosaminoglycans during septic shock: relation to mortality and the antibacterial actions of plasma. Shock 2008, 30:623-627.

11. Sallisalmi M, Tenhunen J, Yang R, Oksala N, Pettila V: Vascular adhesion protein-1 and syndecan-1 in septic shock. Acta Anaesthesiol Scand 2012, 56:316-322.

12. Steppan J, Hofer S, Funke B, Brenner T, Henrich M, Martin E, Weitz J, Hofmann $U$, Weigand MA: Sepsis and major abdominal surgery lead to flaking of the endothelial glycocalyx. J Surg Res 2011, 165:136-141.

13. Kohler M, Kaufmann I, Briegel J, Jacob M, Goeschl J, Rachninger W, Thiel M, Rehm M: The endothelial glycocalyx degenerates with increasing sepsis severity. Crit Care 2011, 15(Suppl 3):P22.

14. Angus DC: The search for effective therapy for sepsis: back to the drawing board? JAMA 2011, 306:2614-2615.

15. Becker BF, Chappell D, Bruegger D, Annecke T, Jacob M: Therapeutic strategies targeting the endothelial glycocalyx: acute deficits, but great potential. Cardiovasc Res 2010, 87:300-310. doi:10.1186/cc11239

Cite this article as: Burke-Gaffney A, Evans TW: Lest we forget the endothelial glycocalyx in sepsis. Critical Care 2012, 16:121. 